Pakistan Journal of Marine Sciences, Vol. 15(2), 131-147, 2006.

\title{
ASSESSMENT OF HEAVY METALS AMONG SUSPENDED PARTICULATES AND DISSOLVED PHASES IN SUEZ CANAL WATER
}

\author{
Mohamed A. Hamed \\ National Institute of Oceanography and Fisheries, Suez, P.O. Box 182
}

\begin{abstract}
The concentrations and distribution of particulate and dissolved heavy metals, viz: $\mathrm{Cu}, \mathrm{Zn}, \mathrm{Pb}, \mathrm{Cd}, \mathrm{Fe}$ and $\mathrm{Mn}$ have been determined seasonally during 2003 in water samples collected from the Suez Canal. The presented data clarifies that the metals exhibited clear differences in their distribution between particulate and dissolved forms. The concentration of particulate heavy metals ranged between 0.09-3.13, 0.57-15.02, 0.18-3.87, 0.02-0.73, 2.74-49.62 and 0.15-5.08 $\mu \mathrm{g} / \mathrm{L}$ for $\mathrm{Cu}, \mathrm{Zn}, \mathrm{Pb}, \mathrm{Cd}, \mathrm{Fe}$ and $\mathrm{Mn}$, respectively. In the same respect, these values for dissolved forms were $0.28-4.12,0.57$ $9.08,0.27-2.50,0.02-1.24,1.94-42.50$ and $0.11-3.65 \mu \mathrm{g} / \mathrm{L}$. The concentrations of particulate metals viz: $\mathrm{Zn}, \mathrm{Pb}, \mathrm{Cd}, \mathrm{Fe}$ and $\mathrm{Mn}$ were high was compared to the dissolved forms. Dissolved copper, rather than particulate, showed the highest percentage of total copper. The particulate forms of $\mathrm{Pb}, \mathrm{Cd}, \mathrm{Fe}$ and $\mathrm{Mn}$ always had higher concentrations than the dissolved forms during the course of study except in the summer season. The northern part of the Suez Canal at Port Said showed mean concentrations of particulate and dissolved $\mathrm{Cu}=1.43$ and $2.10, \mathrm{Zn}=8.61$ and $3.17, \mathrm{~Pb}=1.72$ and $1.23, \mathrm{Cd}=0.35$ and $0.35, \mathrm{Fe}=23.49$ and 15.83 and $\mathrm{Mn}=2.09$ and $1.82 \mu \mathrm{g} / \mathrm{L}$. These high concentrations may be attributed to the greater activities, particularly loading and unloading operations at Port Said harbour, industrial effluents and domestic drainage of Port Said city. In contrast, the Sinai side could be considered as reference site, as it was almost clean, i.e., without harmful outfalls, where $\mathrm{Cu}=0.16$ and $0.56, \mathrm{Zn}=2.14$ and $0.94, \mathrm{~Pb}=6.29$ and $3.44, \mathrm{Cd}=$ 0.055 and $0.088, \mathrm{Fe}=6.29$ and 3.44 and $\mathrm{Mn}=0.56$ and $0.26 \mu \mathrm{g} / \mathrm{L}$ for particulate and dissolved metals respectively.
\end{abstract}

KEYWORDS: Speciation, heavy metals, suspended particulate, dissolved water, sediments, Suez Canal.

\section{INTRODUCTION}

The Suez Canal is actually the only canal linking the Mediterranean Sea with the Red Sea. It is located between Suez and Port Said provinces (lying between longitudes $32^{\circ} 20^{\prime}$ and $32^{\circ} 35^{\prime} \mathrm{E}$ and between latitudes $29^{\circ} 55^{\prime}$ and $31^{\circ} 15^{\prime} \mathrm{N}$ with average length of $164 \mathrm{~km}$ along the major axis). Recent urbanization of the region has led to the situation that the Suez Canal system is suffering considerably from pollution, because it is a big navigation route. Oil pollution from leaks and bilges, domestic pressure, sewage pollution, pesticides and herbicides, agricultural run off, industrial effluent and thermal pollution from power plants are common types of pollution on the Suez Canal, which may directly or indirectly affect the marine life of the canal. Infrastructures established on the canal coasts are landbased sources of these type of pollution. All refuses coming from different sources are discharged directly or indirectly into the Suez Canal. These refuses contain large amount of chemical residues, especially metals and hydrocarbons (Hamed, 1996). In a natural 
aquatic system, metals may be distributed among dissolved, colloidal or particulate fractions as the results of reactions such as precipitation and coprecipitation or complexation with organic or inorganic ligands. In some cases, distributions may depend on the presence of more than one oxidation state for the metal (Leckie and James, 1976). The aim of this work is to assess the distribution of heavy metals among suspended particulates and dissolved phases in the Suez Canal water.

\section{MATERIALS AND METHODS}

\section{Sampling:}

Using Nansen bottles, surface (0-0.5 m depth) water samples were collected seasonally during February, May, August and November 2003 from seventeen stations divided into four sectors (A, B, C and D) between Suez and Port Said provinces (Fig. 1). One more station was located in the southern part of the canal at the Sinai side (sector E).

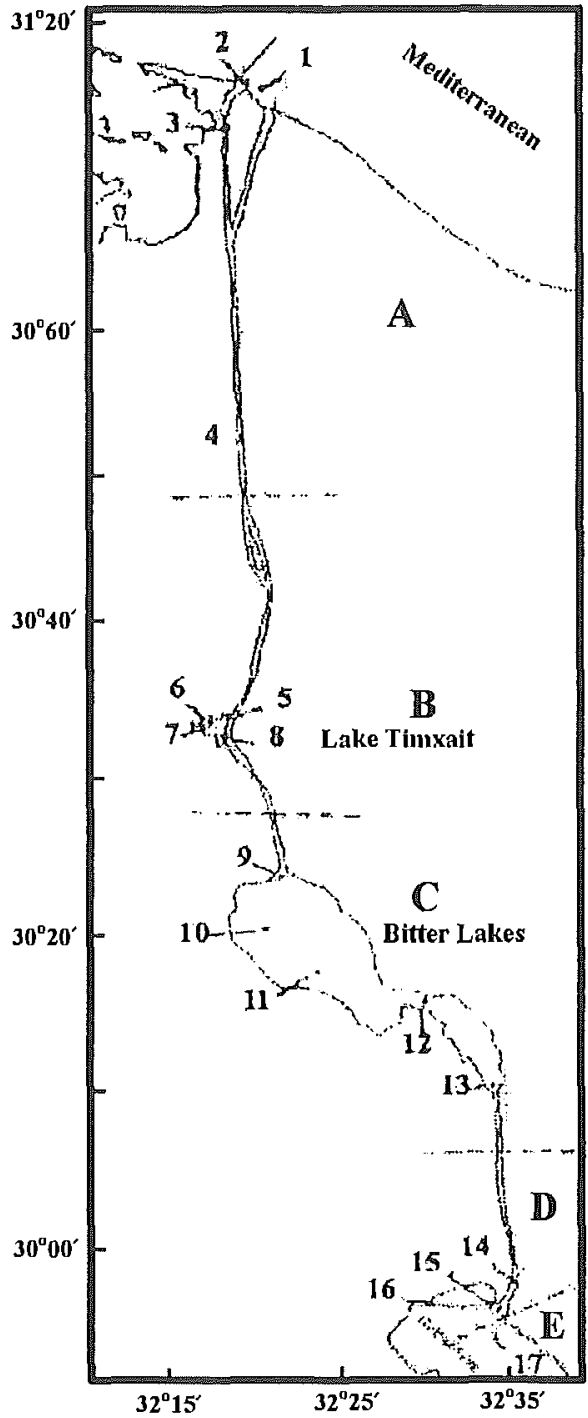

Sector A (Port Said):

1- Port Fouad,

2- Port Said,

3- El-Raswa and

4- El-Qantara.

Sector B (Timsah Lake):

5- Northern entrance of the Lake,

6- infront of Ismailia channel,

7- infront of western lagoon and 8- southern entrance of the lake.

\section{Sector C (Bitter Lakes):}

9- El-Defresoir,

10- Fayed,

11- Fanara,

12- Kabreit and

13- Shandora.

\section{Sector D (Suez Bay):}

14- In front of El-Dersa pond,

15- Southern entrance of the canal 16- ElZeityia (Suez Bay).

Sector E (Sinai side): 17- Ain Moussa. 


\section{Procedures:}

After collecting the samples and filtering them through $0.45 \mu \mathrm{m}$ fiberglass paper (APHA, 1989); each filtered mass was kept for analysis while the filtrate was treated with ammonium pyrralodine dithiocarbamate (APDC) to complex the heavy metals. After shaking well, methyl isobutyl ketone (MIBK) was used for extraction (Brooks et al., 1967). The particulate for each sample was dissolved in $3 \mathrm{~mL}$ of concentrated $\mathrm{HNO}_{3}$ plus $\mathrm{H}_{2} \mathrm{O}_{2}$ (Tesier et al., 1973) and treated as the filtrate. Measurements of the heavy solution for the heavy metals were carried out using Perkin-Elmer 2380 atomic absorption spectrometer.

\section{RESULTS AND DISCUSSION}

\section{Regional distribution of heavy metals:}

The Suez Canal receives various land-based sources of wastes discharged from tankers passing across the canal, domestic wastes, industrial effluents, and fish processing wastes. Moreover, Port Said represents the most industrialized area in the canal at the inlet of the Mediterranean and shows high levels of heavy metals, whereas minimum levels were recorded at Sinai site (Tables 1-6). In the southern part (sector D), Suez showed mean concentrations of particulate and dissolved metals for $\mathrm{Cu}(0.94$ and 1.48), $\mathrm{Zn}$ (7.31 and 2.89), $\mathrm{Pb}$ (1.49 and 1.21), $\mathrm{Cd}$ (0.25 and 0.30), $\mathrm{Fe}$ (19.10 and 7.41) and $\mathrm{Mn}$ (1.38 and 1.37$) \mu \mathrm{g} / \mathrm{L}$, respectively. This part is affected by the invading water coming from Suez Bay where there are many sources of pollution such as domestic drainage from Suez city, industrial wastes of the fertilizer company, power stations and oil refineries. The middle part (sectors B and $\mathrm{C}$ ), near Ismailia, shows slightly higher concentration of $\mathrm{Cu}$ (0.68 and 0.91), $\mathrm{Zn}$ (3.57 and 3.28), $\mathrm{Pb}$ (1.05 and 0.77), $\mathrm{Cd}(0.21$ and 0.18), $\mathrm{Fe}$ (11.11 and 9.2) and $\mathrm{Mn}(1.11$ and 0.96$) \mu \mathrm{g} / \mathrm{L}$, respectively. This is probably the result of agricultural effluents, shipyards of the Suez Canal and sewage discharge. The northern part of the Suez Canal at Port Said (sector A) has mean concentrations of $\mathrm{Cu}(1.43$ and 2.10), $\mathrm{Zn}$ (8.61 and 3.17), $\mathrm{Pb}$ (1.72 and 1.23), $\mathrm{Cd}$ (0.35 and 0.35), Fe (23.49 and 15.83) and Mn (2.09 and 1.82) $\mu \mathrm{g} / \mathrm{L}$, respectively; because this area possesses high sources of activities, such as loading and unloading operations at Port Said harbor and particularly the industrial effluents and domestic drainage of Port Said city. The Sinai side (sector E) might be used as control, as it is almost clean and without harmful outfalls, where the concentrations of $\mathrm{Cu}=0.16$ and $0.56, \mathrm{Zn}=2.14$ and $0.94, \mathrm{~Pb}=6.29$ and $3.44, \mathrm{Cd}=0.055$ and $0.088, \mathrm{Fe}=6.29$ and 3.44 and $\mathrm{Mn}=0.56$ and $0.26 \mu \mathrm{g} / \mathrm{L}$ for particulate and dissolved metals, respectively have been recorded.

\section{Seasonal distribution of heavy metals:}

\section{Copper (Table 1):}

The annual total $\mathrm{Cu}$ ranged from $0.722 \mu \mathrm{g} / \mathrm{L}$ at station 17 to $4.41 \mu \mathrm{g} / \mathrm{L}$ at station 2 , with the annual particulate $\mathrm{Cu}$ measured from $0.16 \mu \mathrm{g} / \mathrm{L}$ at station 17 to 2.04 at station 2 , and the annual dissolved $\mathrm{Cu}$ from $0.56 \mu \mathrm{g} / \mathrm{L}$ at station 17 to $2.57 \mu \mathrm{g} / \mathrm{L}$ at station 3 .The regional variation of copper reached its maximum values $(2.77,1.15$ and $1.61 \mu \mathrm{g} / \mathrm{L}$ ) during the spring for total, particulates and dissolved copper, respectively. Their corresponding minimum values $(1.62,0.69$ and $0.93 \mu \mathrm{g} / \mathrm{L})$ were recorded during the summer. Spring had the maximum average values of total, particulate and dissolved 
Table 1. Seasonal variation of copper $(\mu \mathrm{g} / \mathrm{L})$ in different types of surface seawater of the Suez Camal durimg 2003.

\begin{tabular}{|c|c|c|c|c|c|c|c|c|c|c|c|c|c|c|c|}
\hline \multirow{2}{*}{$\frac{\text { Seasons }}{\text { Stations }}$} & \multicolumn{3}{|c|}{ Winter } & \multicolumn{3}{|c|}{ Spring } & \multicolumn{3}{|c|}{ Summer } & \multicolumn{3}{|c|}{ Autumn } & \multicolumn{3}{|c|}{ Annual mean $\pm S D$} \\
\hline & $T$ & $\mathbf{P}$ & D & $\mathbb{T}$ & $\mathbf{P}$ & D & $\mathbb{T}$ & $\mathbf{P}$ & D & T & $\mathbf{P}$ & D & T & $\mathbb{P}$ & $\mathbb{D}$ \\
\hline 1 & 3.65 & 1.29 & 2.36 & 4.72 & 1.89 & 2.83 & 2.72 & 1.03 & 1.69 & 3.17 & 1.22 & 1.95 & $3.56 \pm 0.74$ & $1.36 \pm 0.32$ & $2.21 \pm 043$ \\
\hline 2 & 3.89 & 2.25 & 1.64 & 7.25 & 3.13 & 4.12 & 2.13 & 0.90 & 1.24 & 4.38 & 1.87 & 2.51 & $4.41 \pm 1.84$ & $2.04 \pm 0.80$ & $2.38 \pm 1.11$ \\
\hline 3 & 4.72 & 1.66 & 3.06 & 5.40 & 1.64 & 3.76 & 3.05 & 1.36 & 1.69 & 2.57 & 0.80 & 1.77 & $3.94 \pm 1.16$ & $1.37 \pm 0.35$ & $2.57 \pm 0.88$ \\
\hline 4 & 2.01 & 0.81 & 1.20 & 2.57 & 1.43 & 1.14 & 1.68 & 0.64 & 1.04 & 2.46 & 0.92 & 1.54 & $2.18 \pm 0.36$ & $0.95 \pm 0.29$ & $1.23 \pm 0.19$ \\
\hline Sector $A$ & & & & & & & & & & & & & $3.52 \pm 0.83$ & $1.43 \pm 0.39$ & $2.10 \pm 0.52$ \\
\hline 5 & 1.51 & 0.54 & 0.97 & 2.36 & 1.21 & 1.05 & 1.25 & 0.85 & 0.40 & 0.87 & 0.59 & 0.28 & $1.5 \pm 0.83$ & $0.80 \pm 0.27$ & $0.67 \pm 0.34$ \\
\hline 6 & 2.14 & 0.60 & 1.54 & $2.70^{\circ}$ & 1.37 & 1.42 & 1.63 & 0.76 & 0.87 & 1.36 & 0.70 & 0.66 & $1.98 \pm 0.55$ & $0.86 \pm 0.30$ & $1.12 \pm 0.37$ \\
\hline 7 & 3.42 & 0.49 & 2.93 & 2.45 & 0.80 & 1.65 & 1.80 & 0.68 & 1.12 & 0.00 & 0.96 & 1.25 & $2.47 \pm 0.60$ & $0.73 \pm 0.17$ & $1.74 \pm 0.72$ \\
\hline 8 & 1.16 & 0.72 & 0.44 & 1.78 & 1.13 & 0.65 & 0.91 & 0.44 & 0.47 & 1.45 & 0.51 & 0.94 & $1.33 \pm 0.33$ & $0.70 \pm 0.27$ & $0.63 \pm 0.20$ \\
\hline Sector B & & & & & & & & & & & & & $1.82 \pm 0.45$ & $0.77 \pm 0.06$ & $1.04 \pm 0.41$ \\
\hline 9 & 1.30 & 0.52 & 0.78 & 0.87 & 0.31 & 0.56 & 0.86 & 0.54 & 0.32 & 1.23 & 0.49 & 0.74 & $1.07 \pm 0.20$ & $0.47 \pm 0.09$ & $0.60 \pm 0.18$ \\
\hline 10 & 1.53 & 1.20 & 0.33 & 1.91 & 0.69 & 1.22 & 1.28 & 0.57 & 0.71 & 1.05 & 0.47 & 0.58 & $1.44 \pm 0.32$ & $0.73 \pm 0.28$ & $0.71 \pm 0.30$ \\
\hline 11 & 1.39 & 0.48 & 0.91 & 1.56 & 0.87 & 0.69 & 1.06 & 0.60 & 0.46 & 1.17 & 0.39 & 0.78 & $1.29 \pm 0.19$ & $0.59 \pm 0.18$ & $0.71 \pm 0.10$ \\
\hline 12 & 2.32 & 0.50 & 1.82 & 2.12 & 0.94 & 1.18 & 1.69 & 0.89 & 0.80 & 1.42 & 0.52 & 0.90 & $1.89 \pm 0.35$ & $0.71 \pm 0.20$ & $1.17 \pm 0.40$ \\
\hline 13 & 1.65 & 0.57 & 1.08 & 1.27 & 0.71 & 0.56 & 1.12 & 0.35 & 0.77 & 0.89 & 0.40 & 0.49 & $1.23 \pm 0.28$ & $0.51 \pm 0.14$ & $0.73 \pm 0.23$ \\
\hline Sector C & & & & & & & & & & & & & $1.38 \pm 0.28$ & $0.60 \pm 0.10$ & $0.78 \pm 0.20$ \\
\hline 14 & 2.86 & 1.32 & 1.54 & 3.21 & 1.13 & 2.08 & 2.48 & 0.95 & 1.53 & 2.79 & 1.31 & 1.48 & $2.85 \pm 0.28$ & $1.18 \pm 0.15$ & $1.66 \pm 0.20$ \\
\hline 15 & 2.54 & 1.20 & 1.34 & 3.79 & 1.35 & 2.44 & 1.71 & 0.59 & 1.12 & 2.33 & 1.08 & 1.25 & $2.59 \pm 0.76$ & $1.06 \pm 0.29$ & $1.54 \pm 0.50$ \\
\hline 16 & 1.92 & 0.60 & 1.32 & 2.24 & 0.77 & 1.47 & 1.54 & 0.52 & 1.02 & 1.68 & 0.48 & 1.20 & $1.85 \pm 0.27$ & $0.59 \pm 0.11$ & $1.25 \pm 0.10$ \\
\hline Sector D & & & & & & & & & & & & & $2.43 \pm 0.42$ & $0.94 \pm 0.25$ & $1.48 \pm 0.13$ \\
\hline Sector E 17 & 0.96 & 0.29 & 0.67 & 0.76 & 0.15 & 0.61 & 0.69 & 0.11 & 0.58 & 0.48 & 0.09 & 0.39 & $0.72 \pm 0.17$ & $0.16 \pm 0.08$ & $0.56 \pm 0.10$ \\
\hline Mean \pm SD & $\begin{array}{r}2.29 \\
\pm 1.08 \\
\end{array}$ & $\begin{array}{c}0.88 \\
\pm 0.52 \\
\end{array}$ & $\begin{array}{c}1.41 \\
\pm 0.78 \\
\end{array}$ & $\begin{array}{r}2.77 \\
\pm 1.70 \\
\end{array}$ & $\begin{array}{c}1.15 \\
\pm 0.68 \\
\end{array}$ & $\begin{array}{c}1.61 \\
\pm 1.10\end{array}$ & $\begin{array}{c}1.62 \\
\pm 0.66 \\
\end{array}$ & $\begin{array}{c}0.69 \\
\pm 0.29 \\
\end{array}$ & $\begin{array}{c}0.93 \\
\pm 0.43 \\
\end{array}$ & $\begin{array}{c}1.85 \\
\pm 1.00 \\
\end{array}$ & $\begin{array}{c}0.75 \\
\pm 0.43 \\
\end{array}$ & $\begin{array}{c}1.08 \\
\pm 0.60 \\
\end{array}$ & $2.13 \pm 0.44$ & $0.87 \pm 0.18$ & $1.26 \pm 0.27$ \\
\hline
\end{tabular}

$T=$ Total, $P=$ Particulate and $D=$ Dissolved

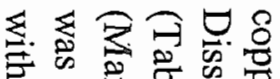

응 훙

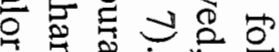

के 25

웅

당 $\approx$

유용

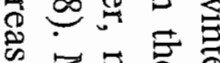

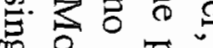

展

두응

흥

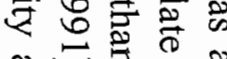

品 8

훙

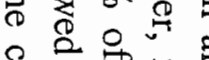

훙

용 율

응

记

․

잉 를.

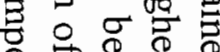

年

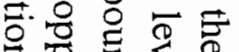

요요

윰ㅇㅇㅁㅇ.

음 $\overrightarrow{0}$

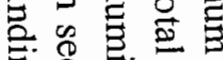

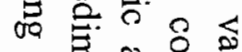

‥ 둥

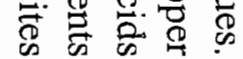


Table 2. Seasonal variation of zinc $(\mu \mathrm{g} / \mathrm{L})$ in different types of surface seawater of the Suez Canal during 2003.

\begin{tabular}{|c|c|c|c|c|c|c|c|c|c|c|c|c|c|c|c|}
\hline \multirow{2}{*}{$\frac{\text { Seasons }}{\text { Stations }}$} & \multicolumn{3}{|c|}{ Winter } & \multicolumn{3}{|c|}{ Spring } & \multicolumn{3}{|c|}{ Summer } & \multicolumn{3}{|c|}{ Autumn } & \multicolumn{3}{|c|}{ Annual mean $\pm \mathrm{SD}$} \\
\hline & $\mathrm{T}$ & $\mathbb{P}$ & $\mathrm{D}$ & $T$ & $\mathbb{P}$ & $\mathbb{D}$ & $\mathbb{T}$ & $\mathbb{P}$ & $\mathbb{D}$ & $\mathbb{T}$ & $\mathbb{P}$ & $\mathbb{D}$ & $T$ & $\mathbb{P}$ & $\mathbb{D}$ \\
\hline 1 & 10.82 & 7.25 & 3.57 & 14.52 & 9.75 & 4.77 & 8.84 & 7.28 & 1.56 & 5.74 & 3.85 & 1.89 & $9.98 \pm 3.68$ & $7.03 \pm 2.42$ & $2.95 \pm 1.50$ \\
\hline 2 & 14.45 & 9.88 & 4.57 & 19.86 & 12.60 & 7.26 & 11.67 & 9.05 & 2.62 & 9.80 & 7.64 & 2.16 & $13.95 \pm 4.38$ & $9.79 \pm 2.09$ & $4.15 \pm 2.32$ \\
\hline 3 & 17.33 & 13.02 & 4.31 & 23.10 & 15.02 & 9.08 & 12.45 & 8.14 & 4.31 & 11.63 & 6.21 & 5.42 & $16.13 \pm 2.32$ & $10.60 \pm 4.11$ & $3.07 \pm 1.70$ \\
\hline 4 & 11.29 & 9.11 & 2.18 & 12.90 & 8.16 & 4.74 & 7.72 & 6.22 & 1.50 & 6.28 & 4.57 & 1.71 & $9.55 \pm 3.07$ & $7.02 \pm 2.03$ & $2.53 \pm 1.50$ \\
\hline Sector A & & & & & & & & & & & & & $12.40 \pm 3.18$ & $8.61 \pm 1.86$ & $3.17 \pm 0.69$ \\
\hline 5 & 7.84 & 4.99 & 2.85 & 5.80 & 3.66 & 2.14 & 4.49 & 2.78 & 1.71 & 4.60 & 3.01 & 1.59 & $5.68 \pm 1.56$ & $3.61 \pm 0.99$ & $2.07 \pm 0.57$ \\
\hline 6 & 9.68 & 8.36 & 1.32 & 6.73 & 4.21 & 2.52 & 7.40 & 4.52 & 2.88 & 3.33 & 2.30 & 1.03 & $6.79 \pm 2.63$ & $4.85 \pm 2.54$ & $1.94 \pm 0.90$ \\
\hline 7 & 10.44 & 7.53 & 2.91 & 8.36 & 5.06 & 3.30 & 5.83 & 3.29 & 2.54 & 4.78 & 2.71 & 2.07 & $7.35 \pm 2.55$ & $4.65 \pm 2.17$ & $2.71 \pm 0.53$ \\
\hline 8 & 6.79 & 5.48 & 1.31 & 4.45 & 3.21 & 1.24 & 3.97 & 2.45 & 1.52 & 2.85 & 1.96 & 0.89 & $4.52 \pm 1.66$ & $3.28 \pm 1.56$ & $1.24 \pm 0.26$ \\
\hline Sector B & & & & & & & & & & & & & $6.09 \pm 1.25$ & $4.10 \pm 0.77$ & $1.99 \pm 0.60$ \\
\hline 9 & 5.58 & 4.85 & 0.73 & 6.40 & 3.82 & 2.58 & 2.31 & 1.62 & 0.69 & 2.63 & 1.79 & 0.84 & $4.23 \pm 2.06$ & $3.02 \pm 1.58$ & $1.21 \pm 0.92$ \\
\hline 10 & 6.38 & 5.12 & 1.26 & 4.47 & 3.65 & 0.82 & 3.46 & 2.38 & 1.08 & 2.98 & 2.26 & 0.72 & $4.32 \pm 1.51$ & $3.35 \pm 1.34$ & $0.97 \pm 0.25$ \\
\hline 11 & 5.23 & 3.98 & 1.25 & 6.14 & 4.58 & 1.56 & 4.21 & 2.99 & 1.22 & 3.75 & 2.45 & 1.30 & $4.83 \pm 1.07$ & $3.50 \pm 0.96$ & $1.33 \pm 0.16$ \\
\hline 12 & 5.69 & 4.30 & 1.39 & 5.02 & 3.14 & 1.88 & 2.85 & 1.46 & 1.39 & 2.12 & 1.43 & 0.69 & $3.92 \pm 1.71$ & $2.58 \pm 1.40$ & $1.34 \pm 0.49$ \\
\hline 13 & 6.14 & 3.95 & 2.19 & 4.68 & 2.89 & 1.79 & 3.62 & 2.33 & 1.29 & 2.74 & 1.55 & 1.19 & $4.30 \pm 1.46$ & $2.68 \pm 1.01$ & $1.62 \pm 0.46$ \\
\hline Sector C & & & & & & & & & & & & & $4.32 \pm 0.33$ & $3.03 \pm 0.40$ & $1.29 \pm 0.24$ \\
\hline 14 & 8.60 & 6.90 & 1.70 & 15.01 & 12.23 & 2.78 & 10.62 & 6.87 & 3.75 & 4.89 & 3.41 & 1.48 & $9.78 \pm 4.22$ & $7.35 \pm 3.64$ & $2.43 \pm 1.05$ \\
\hline 15 & 13.37 & 9.20 & 4.17 & 16.98 & 11.74 & 5.24 & 8.04 & 5.61 & 2.43 & 7.46 & 4.64 & 2.82 & $11.46 \pm 4.54$ & $7.80 \pm 3.28$ & $3.67 \pm 1.29$ \\
\hline 16 & 9.12 & 6.86 & 2.26 & 13.26 & 8.99 & 4.27 & 9.12 & 7.46 & 1.66 & 5.88 & 3.78 & 2.10 & $9.35 \pm 3.02$ & $6.77 \pm 2.19$ & $2.57 \pm 1.16$ \\
\hline Sector D & & & & & & & & & & & & & $10.20 \pm 1.12$ & $7.31 \pm 0.52$ & $2.89 \pm 0.68$ \\
\hline Sector E 17 & 4.32 & 3.06 & 1.26 & 3.95 & 2.61 & 1.34 & 2.16 & 1.59 & 0.57 & 1.89 & 1.31 & 0.58 & $3.08 \pm 1.23$ & $2.14 \pm 0.83$ & $0.94 \pm 0.42$ \\
\hline Mean+SD & 9.00 & 6.70 & 2.31 & 10.10 & 6.78 & 3.37 & 6.40 & 4.47 & 1.92 & 4.90 & 3.23 & 1.68 & $7.22 \pm 3.95$ & $5.04 \pm 2.79$ & $2.06 \pm 0.97$ \\
\hline & \pm 3.62 & \pm 2.62 & \pm 1.22 & \pm 6.08 & \pm 4.13 & \pm 2.27 & \pm 3.35 & \pm 2.58 & \pm 1.03 & \pm 2.71 & \pm 1.75 & 1.15 & & & \\
\hline
\end{tabular}




\section{Zinc (Table 2):}

The annual total Zinc ranged from $3.08 \mu \mathrm{g} / \mathrm{L}$ at station 17 to $16.13 \mu \mathrm{g} / \mathrm{L}$ at station 3 , with the annual particulate $\mathrm{Zn}$ varying from $2.14 \mu \mathrm{g} / \mathrm{L}$ at station 17 to $10.60 \mu \mathrm{g} / \mathrm{L}$ at station 3, while the annual dissolved $\mathrm{Zn}$ varied from $0.94 \mu \mathrm{g} / \mathrm{L}$ at station 17 to $4.15 \mu \mathrm{g} / \mathrm{L}$ at station 2. Regional variation of zinc was maximum $(10.10,6.78$ and $3.37 \mu \mathrm{g} / \mathrm{L})$ during spring for total, particulate and dissolved zinc, respectively. Their respective minimum values of $4.90,3.23$ and $1.68 \mu \mathrm{g} / \mathrm{L}$ were recorded during autumn. Spring exhibited the maximum mean levels for all three forms of zinc and particulate zinc was the dominant form for all seasons comprising about $65.92 \%$ to $74.41 \%$ of the total zinc (Table 7 ). The higher zinc input during spring that bound readily with many organic ligands, particularly in presence of nitrogen or sulfur donor atoms, might explain the higher level particulate zinc over dissolved zinc. Moore (1991) indicated that the binding of zinc to suspended particulates depended on $\mathrm{pH}$ and $\mathrm{E}_{\mathrm{h}}$ conditions, and the input of anthropogenically derived zinc.

\section{Lead (Table 3):}

The annual total $\mathrm{Pb}$ ranged from $0.85 \mu \mathrm{g} / \mathrm{L}$ at station 17 to $3.61 \mu \mathrm{g} / \mathrm{L}$ at station 3 , with the annual particulate $\mathrm{Pb}$ varying from $0.31 \mu \mathrm{g} / \mathrm{L}$ at station 17 to $2.12 \mu \mathrm{g} / \mathrm{L}$ at station 15 , while the annual dissolved $\mathrm{Pb}$ was from $0.54 \mu \mathrm{g} / \mathrm{L}$ at station 17 to $1.52 \mu \mathrm{g} / \mathrm{L}$ at station 2. The regional variation of $\mathrm{Pb}$ reached its maximum values $(2.77,1.83$ and $0.95 \mu \mathrm{g} / \mathrm{L})$ during spring for total, particulates and dissolved $\mathrm{Pb}$, respectively. Their minimum values were $1.91 \mu \mathrm{g} / \mathrm{L}$ for total $\mathrm{Pb}$ during autumn, $0.64 \mu \mathrm{g} / \mathrm{L}$ for particulate $\mathrm{Pb}$ during summer and $0.684 \mu \mathrm{g} / \mathrm{L}$ for dissolved $\mathrm{Pb}$ during winter. The relative decrease in $\mathrm{Pb}$ concentration in winter may be attributed to the relative decrease in the decomposition rate of organic matter with lower temperatures (Aboul-Nagah, 1979 and Hafez, 1982). Table (7) shows that particulate $\mathrm{Pb}$ was dominant at all seasons except during summer, where dissolved $\mathrm{Pb}$ constituted a major portion $(68.97 \%)$ of total lead. Summer was characterized by high salinities accompanied by high chloride ions (Morcos and Riley, 1966), where lead typically desorbed from sediments and suspended solids in estuaries owing to competition with chlorides, thus producing appreciable increases in soluble lead in the water column (Ferrari and Ferrario, 1989).

\section{Cadmium (Table 4):}

The annual total Cd ranged from $0.143 \mu \mathrm{g} / \mathrm{L}$ at station 17 to $0.83 \mu \mathrm{g} / \mathrm{L}$ at station 2 , with the annual particulate Cd varying from $0.055 \mu \mathrm{g} / \mathrm{L}$ at station 17 to $0.44 \mu \mathrm{g} / \mathrm{L}$ at station 2, while the annual dissolved $\mathrm{Cd}$ was measured from $0.088 \mu \mathrm{g} / \mathrm{L}$ at station 17 to $0.40 \mu \mathrm{g} / \mathrm{L}$ at station 15 . The regional variation of $\mathrm{Cd}$ reached its maximum values $(0.92$ and $0.604 \mu \mathrm{g} / \mathrm{L}$ ) during summer for total and dissolved and $0.376 \mu \mathrm{g} / \mathrm{L}$ for particulate $\mathrm{Cd}$ during autumn, while their minimum values of $0.194,0.12$ and $0.076 \mu \mathrm{g} / \mathrm{L}$ where reported during winter for total, particulate and dissolved $\mathrm{Cd}$, respectively. Therefore, particulate $\mathrm{Cd}$ was the dominant form for all seasons but only during the summer season what? and the dissolved $\mathrm{Cd}$ constituted a major percentage $(65.65 \%)$ of total $\mathrm{Cd}$. As salinities increased during the summer, $\mathrm{Cd}$ concentrations increased also. Zirino and Yamamoto (1972) and Abdelmoneim and Fattouh (1994) indicated that at higher salinities, Cd interacted primarily with chloride ions, while the reverse happened for particulate $\mathrm{Cd}$. 
Table 3. Seasonal variation of lead $(\mu \mathrm{g} / \mathrm{L})$ in different types of surface seawater of the Suez Canal during 2003.

\begin{tabular}{|c|c|c|c|c|c|c|c|c|c|c|c|c|c|c|c|}
\hline \multirow{2}{*}{$\frac{\text { Seasons }}{\text { Stations }}$} & \multicolumn{3}{|c|}{ Winter } & \multicolumn{3}{|c|}{ Spring } & \multicolumn{3}{|c|}{ Summer } & \multicolumn{3}{|c|}{ Autumn } & \multicolumn{3}{|c|}{ Annual mean $\pm S D$} \\
\hline & $\mathbb{T}$ & $\mathbb{P}$ & D & $T$ & $P$ & D & $T$ & $\mathbb{P}$ & $\mathrm{D}$ & $T$ & $\mathbf{P}$ & $\mathbb{D}$ & $\mathrm{T}$ & $\mathbf{P}$ & $\mathbb{D}$ \\
\hline 1 & 2.46 & 1.68 & 0.78 & 3.37 & 2.56 & 0.81 & 2.28 & 0.53 & 1.75 & 2.16 & 1.28 & 0.88 & $2.57 \pm 0.55$ & $1.51 \pm 0.85$ & $1.06 \pm 0.47$ \\
\hline 2 & 3.41 & 1.89 & 1.52 & 4.15 & 3.12 & 1.03 & 3.96 & 1.62 & 2.34 & 2.93 & 1.76 & 1.17 & $3.61 \pm 0.55$ & $2.10 \pm 0.69$ & $1.52 \pm 0.59$ \\
\hline 3 & 2.79 & 2.04 & 0.75 & 5.21 & 3.87 & 1.34 & 3.14 & 0.64 & 2.50 & 2.79 & 1.86 & 0.93 & $3.48 \pm 1.16$ & $2.10 \pm 1.33$ & $1.38 \pm 0.77$ \\
\hline 4 & 1.88 & 0.98 & 0.9 & 2.74 & 1.91 & 0.83 & 2.44 & 0.81 & 1.63 & 1.31 & 0.90 & 0.41 & $2.09 \pm 0.63$ & $1.15 \pm 0.51$ & $0.94 \pm 0.51$ \\
\hline Sector A & & & & & & & & & & & & & $2.94 \pm 0.73$ & $1.72 \pm 0.47$ & $1.23 \pm 0.27$ \\
\hline 5 & 1.45 & 0.75 & 0.70 & 2.33 & 1.56 & 0.77 & 0.88 & 0.31 & 0.57 & 1.77 & 1.48 & 0.29 & $1.61 \pm 0.61$ & $1.03 \pm 0.60$ & $0.58 \pm 0.22$ \\
\hline 6 & 1.69 & 1.02 & 0.67 & 2.85 & 1.63 & 1.22 & 1.54 & 0.32 & 1.22 & 1.14 & 0.73 & 0.41 & $1.81 \pm 0.73$ & $0.93 \pm 0.55$ & $0.88 \pm 0.41$ \\
\hline 7 & 2.32 & 1.45 & 0.87 & 3.12 & 1.87 & 1.25 & 2.28 & 0.72 & 1.56 & 2.60 & 1.18 & 1.42 & $2.58 \pm 0.39$ & $1.31 \pm 0.48$ & $1.28 \pm 0.30$ \\
\hline 8 & 1.73 & 0.93 & 0.80 & 1.96 & 1.46 & 0.50 & 1.07 & 0.24 & 0.83 & 1.92 & 1.40 & 0.52 & $1.67 \pm 0.41$ & $1.01 \pm 0.56$ & $0.66 \pm 0.181$ \\
\hline Sector B & & & & & & & & & & & & & $1.92 \pm 0.45$ & $1.07 \pm 0.17$ & $0.85 \pm 0.31$ \\
\hline 9 & 1.81 & 1.30 & 0.51 & $2.18^{\circ}$ & 1.65 & 0.53 & 1.75 & 0.60 & 1.15 & 1.48 & 0.79 & 0.69 & $1.81 \pm 0.29$ & $1.09 \pm 0.48$ & $0.72 \pm 0.30$ \\
\hline 10 & 2.28 & 1.81 & 0.47 & 2.70 & 1.46 & 1.24 & 1.52 & 0.74 & 0.78 & 1.99 & 1.35 & 0.64 & $2.12 \pm 0.50$ & $1.34 \pm 0.45$ & $0.78 \pm 0.33$ \\
\hline 11 & 1.54 & 0.87 & 0.67 & 1.81 & 1.15 & 0.66 & 1.26 & 0.46 & 0.80 & 0.97 & 0.7 & 0.27 & $1.40 \pm 0.36$ & $0.80 \pm 0.29$ & $0.60 \pm 0.23$ \\
\hline 12 & 1.97 & 1.55 & 0.42 & 1.16 & 0.69 & 0.47 & 2.04 & 0.68 & 1.36 & 1.66 & 1.04 & 0.62 & $1.71 \pm 0.40$ & $0.99 \pm 0.41$ & $0.72 \pm 0.44$ \\
\hline 13 & 1.04 & 0.73 & 0.31 & 1.89 & 1.10 & 0.79 & 1.63 & 0.55 & 1.08 & 1.74 & 1.36 & 0.38 & $1.58 \pm 0.37$ & $0.94 \pm 0.36$ & $0.64 \pm 0.36$ \\
\hline Sector C & & & & & & & & & & & & & $1.72 \pm 0.27$ & $1.03 \pm 0.20$ & $0.69 \pm 0.07$ \\
\hline 14 & 2.12 & 1.38 & 0.74 & 3.50 & 2.08 & 1.42 & 3.02 & 0.77 & 2.25 & 2.29 & 1.26 & 1.03 & $2.73 \pm 0.64$ & $1.37 \pm 0.54$ & $1.36 \pm 0.66$ \\
\hline 15 & 2.56 & 2.12 & 0.44 & 4.86 & 3.16 & 1.70 & 2.76 & 0.97 & 1.79 & 3.67 & 2.24 & 1.43 & $3.46 \pm 1.05$ & $2.12 \pm 0.09$ & $1.34 \pm 0.62$ \\
\hline 16 & 1.68 & 1.29 & 0.39 & 2.48 & 1.32 & 1.16 & 1.98 & 0.50 & 1.48 & 1.46 & 0.81 & 0.65 & $1.90 \pm 0.44$ & $0.98 \pm 0.40$ & $0.92 \pm 0.49$ \\
\hline Sector D & & & & & & & & & & & & & $2.70 \pm 0.78$ & $1.49 \pm 0.58$ & $1.21 \pm 0.25$ \\
\hline Sector E 17 & 0.91 & 0.23 & 0.68 & 0.85 & 0.48 & 0.37 & 1.02 & 0.36 & 0.66 & 0.63 & 0.18 & 0.45 & $0.85 \pm 0.16$ & $0.31 \pm 0.14$ & $0.54 \pm 0.15$ \\
\hline Mean \pm SD & $\begin{array}{c}1.98 \\
\pm 0.63\end{array}$ & $\begin{array}{l}1.29 \\
\pm 0.52\end{array}$ & $\begin{array}{l}0.68 \\
\pm 0.28\end{array}$ & $\begin{array}{l}2.77 \\
\pm 1.19\end{array}$ & $\begin{array}{c}1.83 \\
\pm 0.90\end{array}$ & $\begin{array}{c}0.95 \\
\pm 0.38\end{array}$ & $\begin{array}{l}2.03 \\
\pm 0.84\end{array}$ & $\begin{array}{l}0.64 \\
\pm 0.32\end{array}$ & $\begin{array}{c}1.40 \\
\pm 0.60\end{array}$ & $\begin{array}{c}1.91 \\
\pm 0.77\end{array}$ & $\begin{array}{c}1.19 \\
\pm 0.50\end{array}$ & $\begin{array}{c}0.72 \\
\pm 0.37\end{array}$ & $2.03 \pm 0.83$ & $1.12 \pm 0.54$ & $0.90 \pm 0.31$ \\
\hline
\end{tabular}


Table 4. Seasonal variation of cadmium $(\mu \mathrm{g} / \mathrm{L})$ in different types of surface seawater of the Suez Camal during 2003.

\begin{tabular}{|c|c|c|c|c|c|c|c|c|c|c|c|c|c|c|c|}
\hline \multirow{2}{*}{$\begin{array}{l}\text { Seasons } \\
\text { Stations }\end{array}$} & \multicolumn{3}{|c|}{ Winter } & \multicolumn{3}{|c|}{ Spring } & \multicolumn{3}{|c|}{ Summer } & \multicolumn{3}{|c|}{ Autumn } & \multicolumn{3}{|c|}{ Annual mean $\pm S D$} \\
\hline & $\mathrm{T}$ & $\mathbf{P}$ & $\mathbb{D}$ & $T$ & $\mathbf{P}$ & $\mathrm{D}$ & $\mathrm{T}$ & $\mathbb{P}$ & $\mathbb{D}$ & $\mathbb{T}$ & $\mathbb{P}$ & $\mathrm{D}$ & $\mathbb{T}$ & $\mathbf{P}$ & $\mathbb{D}$ \\
\hline 1 & 0.17 & 0.15 & 0.02 & 0.21 & 0.15 & 0.06 & 1.54 & 0.30 & 1.24 & 0.67 & 0.45 & 0.22 & $0.65 \pm 0.64$ & $0.26 \pm 0.14$ & $0.39 \pm 0.58$ \\
\hline 2 & 0.31 & 0.19 & 0.12 & 0.43 & 0.36 & 0.07 & 1.78 & 0.58 & 1.20 & 0.79 & 0.62 & 0.17 & $0.83 \pm 0.67$ & $0.44 \pm 0.20$ & $0.39 \pm 0.54$ \\
\hline 3 & 0.45 & 0.32 & 0.13 & 0.36 & 0.25 & 0.11 & 1.31 & 0.34 & 0.97 & 0.84 & 0.59 & 0.25 & $0.74 \pm 0.43$ & $0.38 \pm 0.15$ & $0.37 \pm 0.41$ \\
\hline 4 & 0.19 & 0.14 & 0.05 & 0.30 & 0.18 & 0.12 & 0.98 & 0.46 & 0.52 & 0.71 & 0.48 & 0.23 & $0.55 \pm 0.37$ & $0.32 \pm 0.18$ & $0.23 \pm 0.21$ \\
\hline Sector A & & & & & & & & & & & & & $0.69 \pm 0.12$ & $0.35 \pm 0.08$ & $0.35 \pm 0.08$ \\
\hline 5 & 0.13 & 0.09 & 0.04 & 0.10 & 0.07 & 0.03 & 0.89 & 0.38 & 0.51 & 0.54 & 0.36 & 0.18 & $0.42 \pm 0.37$ & $0.23 \pm 0.17$ & $0.19 \pm 0.22$ \\
\hline 6 & 0.11 & 0.05 & 0.06 & 0.11 & 0.05 & 0.06 & 0.56 & 0.16 & 0.40 & 0.47 & 0.28 & 0.19 & $0.31 \pm 0.24$ & $0.14 \pm 0.11$ & $0.18 \pm 0.16$ \\
\hline 7 & 0.25 & 0.17 & 0.08 & 0.31 & 0.22 & 0.09 & 1.26 & 0.47 & 0.79 & 0.66 & 0.40 & 0.26 & $0.62 \pm 0.46$ & $0.32 \pm 0.14$ & $0.31 \pm 0.33$ \\
\hline 8 & 0.18 & 0.11 & 0.07 & 0.27 & 0.18 & 0.09 & 0.92 & 0.73 & 0.17 & 0.50 & 0.31 & 0.19 & $0.47 \pm 0.33$ & $0.33 \pm 0.28$ & $0.13 \pm 0.06$ \\
\hline Sector B & & & & & & & & & & & & & $0.46 \pm 0.13$ & $0.26 \pm 0.09$ & $0.20 \pm 0.08$ \\
\hline 9 & 0.12 & 0.02 & 0.10 & 0.19 & 0.12 & 0.07 & 0.75 & 0.13 & 0.62 & 0.38 & 0.29 & 0.09 & $0.36 \pm 0.28$ & $0.14 \pm 0.11$ & $0.22 \pm 0.27$ \\
\hline 10 & 0.15 & 0.08 & 0.07 & 0.09 & 0.04 & 0.05 & 0.67 & 0.23 & 0.44 & 0.36 & 0.21 & 0.15 & $0.32 \pm 0.26$ & $0.14 \pm 0.09$ & $0.18 \pm 0.18$ \\
\hline 11 & 0.10 & 0.06 & 0.04 & 0.07 & 0.03 & 0.04 & 0.48 & 0.18 & 0.30 & 0.59 & 0.33 & 0.26 & $0.31 \pm 0.26$ & $0.15 \pm 0.14$ & $0.16 \pm 0.14$ \\
\hline 12 & 0.26 & 0.16 & 0.10 & 0.20 & 0.11 & 0.09 & 0.39 & 0.14 & 0.25 & 0.61 & 0.50 & 0.11 & $0.37 \pm 0.18$ & $0.23 \pm 0.18$ & $0.14 \pm 0.08$ \\
\hline 13 & 0.09 & 0.04 & 0.05 & 0.26 & 0.12 & 0.14 & 0.50 & 0.12 & 0.38 & 0.32 & 0.23 & 0.09 & $0.29 \pm 0.17$ & $0.13 \pm 0.08$ & $0.17 \pm 0.15$ \\
\hline Sector C & & & & & & & & & & & & & $0.33 \pm 0.03$ & $0.16 \pm 0.04$ & $0.17 \pm 0.03$ \\
\hline 14 & 0.23 & 0.15 & 0.08 & 0.17 & 0.09 & 0.08 & 1.08 & 0.22 & 0.86 & 0.72 & 0.56 & 0.16 & $0.55 \pm 0.43$ & $0.26 \pm 0.21$ & $0.30 \pm 0.38$ \\
\hline 15 & 0.29 & 0.16 & 0.13 & 0.25 & 0.13 & 0.12 & 1.47 & 0.45 & 1.02 & 0.75 & 0.42 & 0.33 & $0.69 \pm 0.57$ & $0.29 \pm 0.17$ & $0.40 \pm 0.42$ \\
\hline 16 & 0.19 & 0.10 & 0.09 & 0.14 & 0.06 & 0.08 & 0.80 & 0.33 & 0.47 & 0.43 & 0.30 & 0.13 & $0.39 \pm 0.30$ & $0.20 \pm 0.14$ & $0.19 \pm 0.19$ \\
\hline Sector D & & & & & & & & & & & & & $0.54 \pm 0.15$ & $0.25 \pm 0.05$ & $0.30 \pm 0.11$ \\
\hline Sector E 17 & 0.08 & 0.02 & 0.06 & 0.07 & 0.03 & 0.04 & 0.24 & 0.11 & 0.13 & 0.18 & 0.06 & 0.12 & $0.14 \pm 0.08$ & $0.06 \pm 0.4$ & $0.09 \pm 0.04$ \\
\hline Mean $\pm S D$ & $\begin{array}{c}0.19 \\
\pm 0.10\end{array}$ & $\begin{array}{c}0.12 \\
\pm 0.08\end{array}$ & $\begin{array}{c}0.076 \\
\pm 0.032\end{array}$ & $\begin{array}{c}0.21 \\
\pm 0.11\end{array}$ & \begin{tabular}{|c|}
0.13 \\
\pm 0.09
\end{tabular} & $\begin{array}{c}0.08 \\
\pm 0.03\end{array}$ & $\begin{array}{c}0.92 \\
\pm 0.44\end{array}$ & $\begin{array}{c}0.31 \\
\pm 0.18\end{array}$ & $\begin{array}{c}0.6 \\
\pm 0.35\end{array}$ & $\begin{array}{c}0.56 \\
\pm 0.18\end{array}$ & $\begin{array}{c}0.38 \\
\pm 0.15\end{array}$ & $\begin{array}{c}0.18 \\
\pm 0.07\end{array}$ & $0.43 \pm 0.21$ & $0.21 \pm 0.10$ & $0.22 \pm 0.10$ \\
\hline
\end{tabular}




\section{Iron (Table 5):}

Annually, the concentration of total iron ranged from $8.98 \mu \mathrm{g} / \mathrm{L}$ at station 17 to 51.0 $\mu \mathrm{g} / \mathrm{L}$ at station 3 and the particulate iron ranged from $6.29 \mu \mathrm{g} / \mathrm{L}$ at station 17 to 30.15 $\mu \mathrm{g} / \mathrm{L}$ at station 2. The dissolved iron ranged from $3.12 \mu \mathrm{g} / \mathrm{L}$ at station 15 to $21.66 \mu \mathrm{g} / \mathrm{L}$ at station 3. According to the regional sense, the maximum Fe concentrations of 30.84 and $19.68 \mu \mathrm{g} / \mathrm{L}$ were recorded during summer for total and dissolved $\mathrm{Fe}$, respectively and 19.63 during winter for particulate Fe. Their minimum values of 17.33 and $5.22 \mu \mathrm{g} / \mathrm{L}$ were recorded during autumn for total and dissolved Fe, respectively and $11.40 \mu \mathrm{g} / \mathrm{L}$ during summer for particulate Fe. Table (7) shows that the particulate forms had higher concentrations than the dissolved forms during the course of study except for the summer season. Only during summer, the dissolved form of Fe constituted a major percentage $(63.81 \%)$ of total $\mathrm{Fe}$. This may be explained by the fact that during the summer, oxygen concentrations in the water-sediment interface decreased to almost zero. This led to reduction of $\mathrm{Fe}^{3+}$ to soluble $\mathrm{Fe}^{2++}$, which was then transported upwards in the water column (Moore, 1991).

\section{Manganese (Table 6):}

Annually, the concentration of total Mn ranged from $0.82 \mu \mathrm{g} / \mathrm{L}$ at station 17 to 4.88 $\mu \mathrm{g} / \mathrm{L}$ at station 3. Particulate $\mathrm{Mn}$ ranged from $0.56 \mu \mathrm{g} / \mathrm{L}$ at stations 10 and 17 to $2.73 \mu \mathrm{g} / \mathrm{L}$ at station 3. Dissolved $\mathrm{Mn}$ ranged from $0.26 \mu \mathrm{g} / \mathrm{L}$ at station 17 to $2.16 \mu \mathrm{g} / \mathrm{L}$ at station 3 . According to the regional sense, the total and particulate $\mathrm{Mn}$ reached their maximum values $(2.78$ and $1.92 \mu \mathrm{g} / \mathrm{L})$ during the winter, while the dissolved form showed maximum values $(1.67 \mu \mathrm{g} / \mathrm{L})$ during summer and autumn. The minimum values of 1.96 and $0.59 \mu \mathrm{g} / \mathrm{L}$ were recorded during spring for total and dissolved $\mathrm{Mn}$, respectively and $0.82 \mu \mathrm{g} / \mathrm{L}$ during summer for particulate Mn. During summer and autumn, dissolved $\mathrm{Mn}$ reached high rates of 67.07 and $60.51 \%$ for the total $\mathrm{Mn}$, respectively (Table 7 ). In summer, the depletion of oxygen at the water-sediment interface caused the reduction of $\mathrm{Mn}^{4+}$ to soluble $\mathrm{Mn}^{2+}$, which was then transported upward in the water column. Oxidation of $\mathrm{Mn}$ occurs much slowly than the oxidation of $\mathrm{Fe}$. As a result, soluble $\mathrm{Mn}$ in water is often supplied almost entirely from in situ reductions in the water column, whereas soluble Fe is supplied by reduction in the sediment (Moore, 1991).

\section{Statistical analysis:}

The presented data show that the metals exhibited clear differences in their distributions between particulate and dissolved forms. Most particulate metals exhibited higher concentration range than that of the dissolved metals. However, both particulate and dissolved forms showed high significant correlations with each other and with total metals (Table 8). This may indicate that the distribution of metals in different phases is governed by interactions of several variables: these include the suspended matter loads of the effluents, their outflow rate concentrations and physical forms of the metals in the effluent, stratification and stability of seawater and the current velocities and patterns (Aboul-Dahab, 1985). Statistical analysis indicated that zinc was highly correlated with manganese and copper, giving high significant positive correlations $(r=0.94,0.92$ and $0.89,0.91)$ for dissolved and particulate metals, respectively. This might be due to adsorption of zinc by hydrous iron oxide (El-Sayed and El-Sayed, 1980). However, the obtained correlation $(r=0.87$ and 0.92$)$ between zinc and copper was due to insolubility 
Table 5. Seasonal variation of iron $(\mu g / L)$ in different types of surface seawater of the Suez Canal during 2003.

\begin{tabular}{|c|c|c|c|c|c|c|c|c|c|c|c|c|c|c|c|}
\hline \multirow{2}{*}{$\begin{array}{l}\text { Seasons } \\
\text { Stations }\end{array}$} & \multicolumn{3}{|c|}{ Winter } & \multicolumn{3}{|c|}{ Spring } & \multicolumn{3}{|c|}{ Summer } & \multicolumn{3}{|c|}{ Autumn } & \multicolumn{3}{|c|}{ Annual mean $\pm S D$} \\
\hline & $T$ & $\mathbb{P}$ & $\mathbb{D}$ & $\mathrm{T}$ & $\mathbb{P}$ & D & $\mathbb{T}$ & $\mathrm{P}$ & D & $\mathrm{T}$ & $P$ & D & $\mathrm{T}$ & $\mathbb{P}$ & $\mathbb{D}$ \\
\hline 1 & 30.21 & 22.90 & 7.31 & 42.50 & 23.70 & 18.80 & 37.80 & 17.59 & 20.21 & 18.25 & 16.05 & 2.20 & $32.19 \pm 10.85$ & $20.06 \pm 3.81$ & $12.13 \pm 8.79$ \\
\hline 2 & 48.56 & 31.87 & 16.69 & 63.24 & 49.62 & 13.42 & 45.07 & 11.39 & 33.68 & 32.46 & 27.70 & 4.76 & $47.33 \pm 12.66$ & $30.15 \pm 15.71$ & $17.14 \pm 12.12$ \\
\hline 3 & 51.74 & 40.62 & 11.12 & 57.31 & 40.43 & 16.88 & 58.27 & 15.77 & 42.50 & 36.68 & 20.56 & 16.12 & $51.00 \pm 9.97$ & $29.35 \pm 13.06$ & $21.66 \pm 3.00$ \\
\hline 4 & 26.30 & 16.11 & 10.19 & 28.77 & 18.92 & 9.85 & 29.92 & 7.80 & 22.12 & 21.85 & 14.42 & 7.43 & $26.71 \pm 3.57$ & $14.31 \pm 4.72$ & $12.40 \pm 6.60$ \\
\hline Sector A & & & & & & & & & & & & & $39.31 \pm 11.70$ & $23.49 \pm 7.62$ & $15.83 \pm 4.52$ \\
\hline 5 & 24.75 & 15.70 & 9.05 & 26.30 & 14.30 & 12.00 & 31.56 & 13.19 & 18.37 & 15.90 & 8.51 & 7.39 & $24.63 \pm 6.51$ & $12.93 \pm 3.12$ & $11.70 \pm 4.84$ \\
\hline 6 & 37.24 & 26.16 & 11.08 & 33.07 & 27.51 & 5.56 & 35.44 & 10.40 & 25.04 & 16.32 & 10.85 & 5.47 & $30.52 \pm 9.62$ & $10.73 \pm 9.38$ & $11.79 \pm 9.22$ \\
\hline 7 & 28.85 & 24.48 & 4.37 & 25.52 & 3.72 & 21.8 & 28.30 & 11.55 & 16.75 & 19.77 & 15.48 & 4.29 & $25.61 \pm 4.16$ & $13.81 \pm 8.63$ & $11.80 \pm 8.78$ \\
\hline 8 & 20.62 & 17.33 & 3.29 & 22.45 & 13.16 & 9.29 & 23.83 & 11.53 & 12.30 & 7.14 & 6.22 & 0.92 & $18.51 \pm 7.69$ & $12.06 \pm 4.60$ & $6.45 \pm 5.26$ \\
\hline Sector B & & & & & & & & & & & & & $24.82 \pm 4.93$ & $12.38 \pm 1.31$ & $10.44 \pm 2.66$ \\
\hline 9 & 17.33 & 12.67 & 4.66 & 15.88 & 8.36 & 7.52 & 22.75 & 7.27 & 15.48 & 11.22 & 8.90 & 2.32 & $16.80 \pm 4.75$ & $9.30 \pm 2.35$ & $7.50 \pm 5.73$ \\
\hline 10 & 21.42 & 13.24 & 8.18 & 26.51 & 11.47 & 15.06 & 28.17 & 10.66 & 17.51 & 21.07 & 12.19 & 8.88 & $24.29 \pm 3.59$ & $11.96 \pm 1.07$ & $12.41 \pm 4.60$ \\
\hline 11 & 18.78 & 10.30 & 8.48 & 11.20 & 8.75 & 2.45 & 27.36 & 12.57 & 14.79 & 9.17 & 2.74 & 6.43 & $16.63 \pm 8.24$ & $8.59 \pm 4.20$ & $8.04 \pm 5.15$ \\
\hline 12 & 15.51 & 9.26 & 6.25 & 19.27 & 12.83 & 6.44 & 20.66 & 9.80 & 11.85 & 15.98 & 11.83 & 4.15 & $17.86 \pm 2.51$ & $10.93 \pm 1.68$ & $7.17 \pm 3.29$ \\
\hline 13 & 11.60 & 7.54 & 4.06 & 14.64 & 10.41 & 4.23 & 19.14 & 10.19 & 8.95 & 6.81 & 5.27 & 1.54 & $13.05 \pm 5.18$ & $8.35 \pm 2.43$ & $4.70 \pm 3.05$ \\
\hline Sector C & & & & & & & & & & & & & $17.73 \pm 4.09$ & $9.83 \pm 1.56$ & $7.69 \pm 2.80$ \\
\hline 14 & 35.16 & 28.12 & 7.04 & 37.05 & 34.23 & 2.82 & 33.42 & 9.78 & 23.64 & 16.58 & 9.25 & 7.33 & $30.55 \pm 9.43$ & $20.35 \pm 12.75$ & $10.21 \pm 9.19$ \\
\hline 15 & 44.22 & 30.15 & 14.07 & 28.15 & 22.19 & 5.96 & 40.5 & 13.25 & 27.25 & 22.30 & 17.09 & 5.21 & $33.79 \pm 10.29$ & $20.67 \pm 7.30$ & $3.12 \pm 10.24$ \\
\hline 16 & 29.47 & 20.97 & 8.50 & 24.96 & 17.44 & 7.52 & 29.24 & 12.04 & 17.20 & 17.02 & 14.65 & 2.37 & $25.17 \pm 5.82$ & $16.27 \pm 3.83$ & $8.90 \pm 6.15$ \\
\hline Sector D & & & & & & & & & & & & & $29.84 \pm 4.35$ & $19.10 \pm 2.45$ & $7.41 \pm 3.77$ \\
\hline Sector E 17 & 9.12 & 6.32 & 2.80 & 7.91 & 5.77 & 2.14 & 12.84 & 8.96 & 6.88 & 6.06 & 4.12 & 1.94 & $8.98 \pm 2.86$ & $6.29 \pm 2.01$ & $3.44 \pm 2.32$ \\
\hline Mean \pm SD & $\begin{array}{l}27.70 \\
\pm 12.46\end{array}$ & $\begin{array}{l}19.63 \\
\pm 9.66\end{array}$ & $\begin{array}{c}8.07 \\
\pm 3.80\end{array}$ & $\begin{array}{c}28.51 \\
\pm 14.90\end{array}$ & $\begin{array}{c}18.99 \\
\pm 12.72\end{array}$ & $\begin{array}{l}9.51 \\
\pm 5.95\end{array}$ & $\begin{array}{c}30.84 \\
\pm 10.70\end{array}$ & $\begin{array}{l}11.40 \\
\pm 2.62\end{array}$ & $\begin{array}{l}19.68 \\
\pm 8.95\end{array}$ & $\begin{array}{l}17.33 \\
\pm 8.39\end{array}$ & $\begin{array}{l}12.11 \\
\pm 6.35\end{array}$ & $\begin{array}{c}5.22 \\
\pm 3.70\end{array}$ & $26.10 \pm 5.99$ & $15.53 \pm 4.38$ & $10.62 \pm 6.32$ \\
\hline
\end{tabular}


Table 6. Seasonal variation of manganese $(\mu \mathrm{g} / \mathrm{L})$ in different types of surface seawater of the Suez Canal during 2003.

\begin{tabular}{|c|c|c|c|c|c|c|c|c|c|c|c|c|c|c|c|}
\hline \multirow{2}{*}{$\begin{array}{l}\text { Seasons } \\
\text { Stations }\end{array}$} & \multicolumn{3}{|c|}{ Winter } & \multicolumn{3}{|c|}{ Spring } & \multicolumn{3}{|c|}{ Summer } & \multicolumn{3}{|c|}{ Autumn } & \multicolumn{3}{|c|}{ Annual mean $\pm \mathrm{SD}$} \\
\hline & $\mathbb{T}$ & $\mathbb{P}$ & D & $\mathbb{T}$ & $\mathbb{P}$ & D & $\mathrm{T}$ & $P$ & $\mathbb{D}$ & $T$ & $\mathbb{P}$ & $\mathbb{D}$ & $T$ & $\mathbb{P}$ & $\mathbb{D}$ \\
\hline 1 & 3.75 & 2.44 & 1.31 & 2.86 & 1.89 & 0.97 & 3.65 & 1.34 & 2.31 & 2.26 & 0.46 & 1.8 & $3.13 \pm 0.70$ & $1.53 \pm 0.84$ & $1.60 \pm 0.58$ \\
\hline 2 & 5.17 & 3.65 & 1.52 & 4.63 & 3.24 & 1.39 & 4.12 & 1.04 & 3.08 & 3.87 & 1.34 & 2.53 & $4.45 \pm 0.58$ & $2.32 \pm 1.32$ & $2.13 \pm 1.80$ \\
\hline 3 & 6.24 & 5.08 & 1.16 & 3.21 & 2.75 & 0.46 & 4.76 & 1.41 & 3.35 & 5.32 & 1.67 & 3.65 & $4.88 \pm 1.27$ & $2.73 \pm 1.67$ & $2.16 \pm 1.58$ \\
\hline 4 & 3.93 & 2.30 & 1.63 & 2.04 & 1.48 & 0.56 & 2.47 & 0.59 & 1.88 & 4.25 & 2.77 & 1.48 & $3.17 \pm 1.08$ & $1.79 \pm 0.96$ & $1.39 \pm 0.58$ \\
\hline Sector A & & & & & & & & & & & & & $3.91 \pm 0.89$ & $2.09 \pm 0.54$ & $1.82 \pm 0.39$ \\
\hline 5 & 2.18 & 1.56 & 0.62 & 2.65 & 2.10 & 0.55 & 1.08 & 0.36 & 0.72 & 3.46 & 1.44 & 2.02 & $2.34 \pm 0.99$ & $1.37 \pm 0.73$ & $0.98 \pm 0.70$ \\
\hline 6 & 2.56 & 1.75 & 0.81 & 1.78 & 1.37 & 0.41 & 2.14 & 0.77 & 1.37 & 2.83 & 0.88 & 1.95 & $2.33 \pm 0.46$ & $1.19 \pm 0.45$ & $1.14 \pm 0.67$ \\
\hline 7 & 3.07 & 1.98 & 1.09 & 2.19 & 1.51 & 0.68 & 1.95 & 0.29 & 1.66 & 3.18 & 1.87 & 1.31 & $2.60 \pm 0.62$ & $1.41 \pm 0.77$ & \\
\hline 8 & 1.81 & 0.96 & 0.85 & 2.56 & 1.73 & 0.83 & 1.42 & 0.48 & 0.94 & 2.31 & 0.86 & 1.45 & $1.19 \pm 0.41$ & $1.00 \pm 0.52$ & $1.02 \pm 0.29$ \\
\hline Sector B & & & & & & & & & & & & & $2.12 \pm 0.63$ & $1.24 \pm 0.19$ & $1.08 \pm 0.10$ \\
\hline 9 & 1.44 & 0.87 & 0.57 & 0.89 & 0.42 & 0.47 & 2.23 & 1.21 & 1.02 & 2.91 & 0.81 & 2.10 & $1.87 \pm 0.89$ & $0.83 \pm 0.32$ & $1.04 \pm 0.75$ \\
\hline 10 & 1.28 & 0.76 & 0.52 & 1.08 & 0.40 & 0.68 & 1.59 & 0.63 & 0.96 & 1.75 & 0.43 & 1.32 & $1.43 \pm 0.30$ & $0.56 \pm 0.17$ & $0.87 \pm 0.35$ \\
\hline 11 & 2.04 & 1.73 & 0.31 & 1.25 & 1.02 & 0.23 & 3.11 & 1.14 & 1.97 & 2.56 & 1.70 & 0.86 & $2.24 \pm 0.79$ & $1.40 \pm 0.37$ & $0.84 \pm 0.80$ \\
\hline 12 & 1.79 & 1.21 & 0.58 & 0.48 & 0.33 & 0.15 & 2.01 & 0.76 & 1.25 & 1.17 & 0.67 & 0.50 & $1.36 \pm 0.69$ & $0.74 \pm 0.36$ & $0.62 \pm 0.46$ \\
\hline 13 & 1.66 & 0.93 & 0.73 & 1.46 & 0.80 & 0.66 & 0.98 & 0.23 & 0.75 & 2.15 & 0.75 & 1.40 & $1.56 \pm 0.48$ & $1.38 \pm 0.57$ & $0.89 \pm 0.35$ \\
\hline Sector $\mathrm{C}$ & & & & & & & & & & & & & $1.69 \pm 0.36$ & $0.98 \pm 0.38$ & $0.85 \pm 0.15$ \\
\hline 14 & 2.14 & 1.45 & 0.69 & 1.80 & 1.45 & 0.35 & 2.98 & 0.34 & 2.64 & 3.29 & 1.22 & 2.07 & $2.55 \pm 0.70$ & $1.12 \pm 0.53$ & $1.44 \pm 1.09$ \\
\hline 15 & 4.25 & 3.12 & 1.13 & 2.32 & 1.62 & 0.70 & 3.76 & 1.58 & 2.18 & 3.44 & 0.53 & 2.91 & $3.44 \pm 0.82$ & $1.71 \pm 1.07$ & $1.73 \pm 1.00$ \\
\hline 16 & 2.71 & 2.04 & 0.67 & 1.66 & 0.90 & 0.76 & 3.25 & 1.55 & 1.70 & 1.36 & 0.70 & 0.66 & $2.25 \pm 0.86$ & $1.30 \pm 0.61$ & $0.95 \pm 0.50$ \\
\hline Sector D & & & & & & & & & & & & & $2.75 \pm 0.62$ & $1.38 \pm 0.30$ & $1.37 \pm 0.39$ \\
\hline Sector E 17 & 1.17 & 0.81 & 0.36 & 0.39 & 0.28 & 0.11 & 0.75 & 0.15 & 0.60 & 0.96 & 0.54 & 0.42 & $0.82 \pm 0.33$ & $0.56 \pm 0.22$ & $0.26 \pm 0.15$ \\
\hline \multirow[t]{2}{*}{ Mean \pm SD } & 2.78 & 1.92 & 0.86 & 1.96 & 1.37 & 0.59 & 2.49 & 0.82 & 1.67 & 2.76 & 1.10 & 1.67 & \multirow[t]{2}{*}{$2.26 \pm 1.16$} & \multirow[t]{2}{*}{$1.25 \pm 0.56$} & \multirow[t]{2}{*}{$1.08 \pm 0.58$} \\
\hline & \pm 1.44 & \pm 11.55 & \pm 0.39 & \pm 1.06 & \pm 0.84 & \pm 0.31 & \pm 1.17 & \pm 0.49 & \pm 0.84 & \pm 1.14 & \pm 0.63 & \pm 0.86 & & & \\
\hline
\end{tabular}


Table 7. The percentage of particulate (P) and dissolved (D) metals to the total metals.

\begin{tabular}{|c|c|c|c|c|c|}
\hline \multirow{2}{*}{\multicolumn{2}{|c|}{$\begin{array}{ll} & \text { Seasons } \\
\text { Metals } & \\
\end{array}$}} & Winter & Spring & Summer & Autumn \\
\hline & & & & & \\
\hline \multirow{2}{*}{$\mathrm{Cu}$} & $\mathrm{P}$ & 38.65 & 41.52 & 42.59 & 40.54 \\
\hline & $\mathrm{D}$ & 61.57 & 58.12 & 57.41 & 58.38 \\
\hline \multirow{2}{*}{$\mathbb{Z}_{\mathbf{n}}$} & $P$ & 74.41 & 67.13 & 69.84 & 65.92 \\
\hline & $\mathrm{D}$ & 25.66 & 33.37 & 30.05 & 34.29 \\
\hline \multirow{2}{*}{$\mathbf{P b}$} & $P$ & $65: 4$ & 66.06 & 31.53 & 62.57 \\
\hline & $\mathrm{D}$ & 34.55 & 34.29 & 68.97 & 37.7 \\
\hline \multirow{2}{*}{$\mathrm{Cd}$} & $\mathbf{P}$ & 61.86 & 61.43 & 34.13 & 67.14 \\
\hline & $\mathrm{D}$ & 39.18 & 37.62 & 65.65 & 32.86 \\
\hline \multirow{2}{*}{$\mathbf{F e}$} & $P$ & 70.87 & 66.61 & 36.96 & 69.88 \\
\hline & $\mathrm{D}$ & 29.13 & 33.36 & 63.81 & 30.30 \\
\hline \multirow{2}{*}{ Mn } & $\mathbf{P}$ & 69.07 & 69.9 & 32.93 & 39.86 \\
\hline & $\mathrm{D}$ & 30.93 & 30.10 & 67.07 & 60.51 \\
\hline
\end{tabular}

In the present work, the concentrations of heavy metals studied agreed with those found in the literature. Table (9) shows that $\mathrm{Cu}$ and $\mathrm{Zn}$ concentrations in the present study were lower than those reported by Fahmy (1981) and Abo-El-Khair (1993) while Pb was higher than that of Abo-El-Khair (1993)and Cd fell within the range elsewhere.

Table 8. Correlation (r) between total (T), particulate (P) and dissolved (D) forms of metals in water of the area area of investigation during 2003.

\begin{tabular}{|c|c|c|c|c|c|c|c|c|c|c|c|c|c|c|c|c|c|c|}
\hline Metals & \multicolumn{3}{|c|}{$\mathrm{Cu}$} & \multicolumn{3}{|c|}{$\mathbb{Z n}$} & \multicolumn{3}{|c|}{$\mathbf{P b}$} & \multicolumn{3}{|c|}{$\mathrm{Cd}$} & \multicolumn{3}{|c|}{$\mathbf{F e}$} & \multicolumn{3}{|c|}{ Mn } \\
\hline $\begin{array}{l}\text { forms } \\
\text { of metals }\end{array}$ & $\mathbb{T}$ & $\mathbf{P}$ & D & $\mathbf{T}$ & $\mathbf{P}$ & D & $\mathbf{T}$ & $\mathbf{P}$ & D & $\mathbb{T}$ & $\mathbb{P}$ & D & $\mathbf{T}$ & $\mathbf{P}$ & D & $\mathrm{T}$ & $\mathbf{P}$ & D \\
\hline Total & 1 & & & 1 & & & 1 & & & 1 & & & 1 & & & 1 & & \\
\hline Particulate & 0.99 & 1 & & 0.99 & 1 & & 0.99 & 1 & & 0.98 & 1 & & 0.99 & 1 & & 0.99 & 1 & \\
\hline Dissolved & 0.99 & 0.96 & 1 & 0.98 & 0.98 & 1 & 0.97 & 0.93 & 1 & 0.97 & 0.92 & 1 & 0.97 & 0.95 & 1 & 0.98 & 0.97 & 1 \\
\hline
\end{tabular}


Table 9. Comparison between the concentrations of $\mathrm{Cu}, \mathrm{Zn}, \mathbb{P b}, \mathrm{Cd}, \mathrm{Fe}$ and $\mathrm{Mn}(\mu \mathrm{g} / \mathrm{L}$ ) in different ecosystems and the present study.

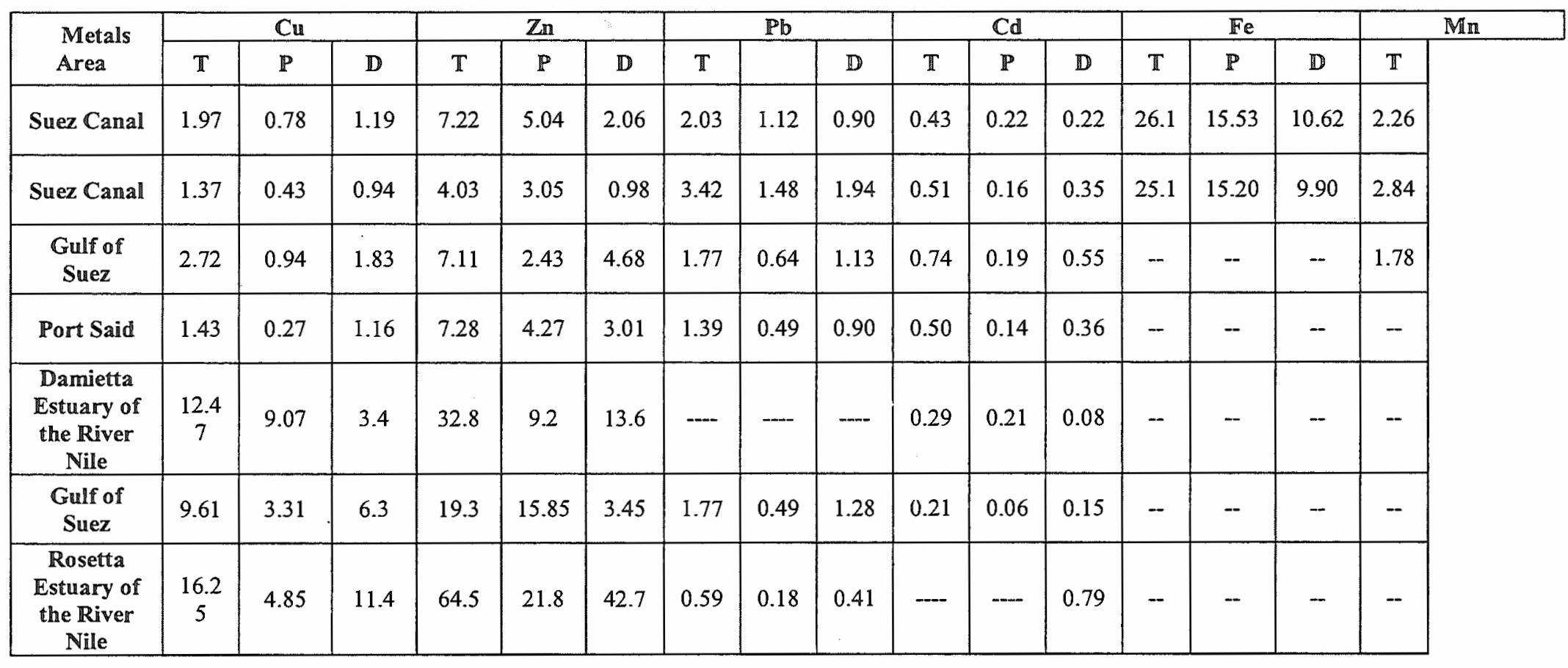


Table 10. Heavy metal concentrations $(\mu \mathrm{g} / \mathrm{g})$ in sediments collected from the area of investigation during 2003.

\begin{tabular}{|c|c|c|c|c|c|c|}
\hline Stations & $\mathrm{CU}$ & $\mathbb{Z N}$ & $\mathbb{P b}$ & $\mathrm{Cd}$ & $\operatorname{Fe} \times 10^{3}$ & $\mathbb{M n}$ \\
\hline 1 & 38.67 & 44.54 & 29.12 & 4.63 & 2.66 & 485.15 \\
\hline 2 & 54.48 & 75.52 & 32.31 & 3.24 & 2.78 & 406.60 \\
\hline 3 & 63.30 & 57.63 & 46.44 & 5.12 & 2.89 & 521.75 \\
\hline 4 & 35.02 & 40.16 & 38.29 & 2.89 & 2.81 & 389.03 \\
\hline Sector A & $47.87 \pm 13.31$ & $54.46 \pm 15.88$ & $36.54 \pm 7.62$ & $2.97 \pm 1.07$ & $2.79 \pm 0.1$ & $450.63 \pm 63.20$ \\
\hline 5 & 25.11 & 36.73 & 27.56 & 1.72 & 2.55 & 298.25 \\
\hline 6 & 31.21 & 37.41 & 21.78 & 2.56 & 2.42 & 336.10 \\
\hline 7 & 42.64 & 48.79 & 36.89 & 3.25 & 2.73 & 376.85 \\
\hline 8 & 29.52 & 32.37 & 15.24 & 1.43 & 2.61 & 352.90 \\
\hline Sector $\mathbb{B}$ & $32.12 \pm 7.47$ & $38.83 \pm 7.01$ & $25.37 \pm 9.18$ & $2.24 \pm 0.83$ & $2.58 \pm 0.13$ & $341.03 \pm 33.06$ \\
\hline 9 & 18.4 & 15.35 & 24.85 & 1.85 & 2.22 & 256.45 \\
\hline 10 & 21.75 & 33.88 & 25.04 & 1.58 & 2.48 & 324.25 \\
\hline 11 & 10.68 & 25.70 & 21.66 & 2.24 & 2.16 & 289.93 \\
\hline 12 & 14.36 & 19.96 & 28.45 & 2.78 & 2.36 & 266.27 \\
\hline 13 & 17.14 & 13.22 & 19.20 & 2.97 & 2.31 & 275.83 \\
\hline Sector C & $15.98 \pm 2.67$ & $21.62 \pm 8.36$ & $23.84 \pm 3.54$ & $2.28 \pm 0.59$ & $2.31 \pm 0.12$ & $282.55 \pm 26.39$ \\
\hline 14 & 42.13 & 47.15 & 34.72 & 3.04 & 2.79 & 267.08 \\
\hline 15 & 49.41 & 56.90 & 43.37 & 3.65 & 2.85 & 412.46 \\
\hline 16 & 36.95 & 31.46 & 26.50 & 2.78 & 2.63 & 386.12 \\
\hline Sector D & $42.83 \pm 6.26$ & $45.17 \pm 12.84$ & $34.86 \pm 8.44$ & $3.16 \pm 0.45$ & $2.76 \pm 0.11$ & $355.22 \pm 77.46$ \\
\hline 17 (Sector E) & 12.31 & 20.44 & 18.10 & 1.85 & 2.12 & 243.60 \\
\hline
\end{tabular}

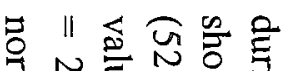

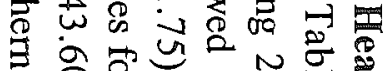

$F N \stackrel{0}{\sigma}$

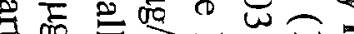

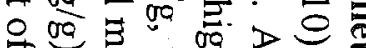

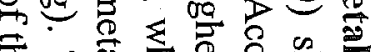

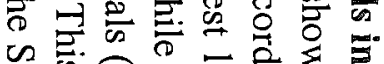

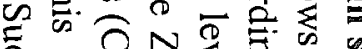

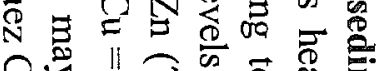

०थ

き灾出灾完

क्ष

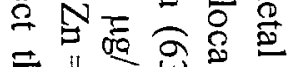

है

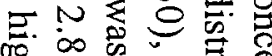

里

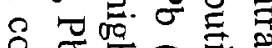

॥亘宫

它舟品吊

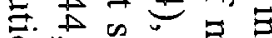

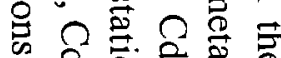

의

$\rightarrow-N=\stackrel{0}{0}$

范的志声

定

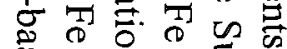

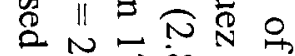

깅

․

웅희

क

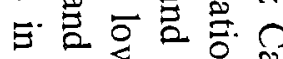

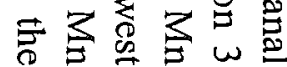


Table (10) shows that mean concentrations of heavy metals in the sediments in the Suez Canal were somewhat higher in the northern parts (sector A) with decreases in the following order of the southern part (sector D) $>$ the middle part (sectors B and C) $>$ Sinai side (sector E). It is clear that all metals studied showed a common trend of increase toward the northern part of the Suez Canal especially at station 3 that receives quantities of municipal and industrial wastewaters from many outlets as well as from ships crossing the canal to or from the Mediterranean. All these sources can have an immediate effect on metal concentrations of marine sediments (Mohapatra, 1988). Rajkumar et al. (1992) reported that the metals entering marine coastal areas became associated with sediments, especially the smaller particles, with the sediments in the northern part of the Suez Canal comprised of light gray muddy sands of the Nile origin (UNEP, 1997). Nicholsen and Moore, (1981) observed that a major portion of metal inputs entered the marine systems in the form of metals rich of finally divided particulate matter, which precipitated on encountering high electrolyte concentrations. The relatively high concentrations of heavy metals in the sediments at the southern part of the Suez Canal may be explained by the fact that this part is affected by large amount of invading water coming from the Suez Bay, which is largely loaded with oil and industrial effluents.

\section{CONCLUSION AND RECOMMENDATIONS}

From the present study, it can be concluded that heavy metals exhibited clear variations in their distribution between the particulate and dissolved forms, where the concentration of the particulate forms were higher than the dissolved phases. Land-based activities are the main pollution sources in the investigated area. Concentrations of particulate and dissolved heavy metals viz: $\mathrm{Cu}, \mathrm{Zn}, \mathrm{Pb}, \mathrm{Cd}, \mathrm{Fe}$ and $\mathrm{Mn}$ the Suez Canal can be divided into three main areas, the southern part (sector D) that is affected by the invading water coming from the Suez Bay, where there are many sources of pollution. The middle part of the Canal, including Bitter Lakes and Lake Timsah (sectors B and C) that are affected by agricultural effluents and sewage discharge. The northern part (sector A), possesses high sources of activities. Thus it is obligatory for the cities within boundaries of the Suez Canal and national organizations of environmental protection to introduce control management plans to protect the Suez Canal. This might include the supply of treatment units to the industrial companies and to all kinds of vehicles, as well as prevention of discharging waste-waters in the area. Moreover, rapid, effective techniques should be applied to the disposed effluents.

\section{REFERENCES}

Abdelmoneim, M.A. and F.A. Fattouh, 1994. Distribution of some heavy metals in coastal recreational areas off Port-Said. The Bulletin of the High Institute of Public Health, XXIV(2): 315-338.

Abo-El-Khair, E.M.H. 1993. Chemical studies on the Mediterranean coastal waters in front of the Rosetta mouth of the Nile. M.Sc. thesis, Fac. Sci., Alexandra Univ., pp. 243. 
Aboul-Dahab, O.M.T. 1985. Chemical cycle of inorganic pollutants in the ecosystem west of Alexandria between Anfoushy and Agamy, Ph.D. thesis Fac. Sci., Alexendra Univ., pp. 338.

Aboul-Nagah, W.M. 1979. The occurrence and distribution of some trace metals in the Mediterranean waters off the coast of Alexandria and their effect on the water productivity, M.Sc. thesis, Fac. Sci., Alexandra Univ., 155 p.

APHA, AWWA and WOCF 1989. Standard methods for the examination of water and wastewater, $17^{\text {th }}$ ed., EPHA, Washington, USA.

Brooks, R.R., B.J. Persely, and I.R. Kaplan, 1967. APDC-MIBK extraction system for the determination of trace elements in saline water by atomic absorption spectrometry, Talanta, 14(7): 809-816.

El-Sayed, M.A. and M. El-Sayed, 1980. Levels of heavy metals in the surface water of a semi-enclosed basin along the Egyptian Mediterranean coast. Journal Etudes Pollution, Cagliari CIESM, 5: 223-228.

El-Moselhy, Kh.M. 1993. Studies of some heavy metals level in some economic fishes in the Suez Gulf, M.Sc. thesis, Fac. Sci., Mans. Univ., pp. 122.

Fahmy, M.A. 1981. Seasonal distribution of heavy metals in the Damietta branch of the Nile. M.Sc. Thesis, Faculty of Science, University of Alexandria, Egypt.

Ferrari, G.M. and P. Ferrario, 1989, Behaviour of $\mathrm{Cd}, \mathrm{Pb}$ and $\mathrm{Cu}$ in the marine deltaic area of the Po River (North Adriatic sea). Water, Air and soil pollution, 43: 323-343.

Hafez, H.H. 1982. Distribution of heavy metals in Lake Mariut and Nozha Hydrodrome and their accumulation in fish, M.Sc. thesis, Fac. Sci., Alexandria Univ., pp. 177.

Hamed, M.A. 1996. Determination of some micro-element in the aquatic ecosystem and their relation to the efficiency of aquatic life, Ph.D. thesis, Fac. Sci., Mans. Univ., pp. 136 .

Hamed, M.A. 1999. Occurrence and distribution of some heavy metals in water and some fishes in the Gulf of Suez, Egypt. Journal of Aquatic Biology and Fisheries, 3(3): 117-135.

Johnson, C.A. 1986. The regulation of trace element concentrations in River and estuarine water contaminated with acid mine drainage. The adsorption of $\mathrm{Cu}$ and $\mathrm{Zn}$ on amorphous Fe oxyhydroxides, Geochimica et Cosmochimica Acta, 50: 2433-8.

Leckie, J.O. and R.O. James, 1976. Control mechanisms for trace metals in natural waters. In: A.J. Rubin, ed. Aqueous - Environmental Chemistry of metals, Ann Arbor Science Publishers, Ann Arbor, MI, pp. 1-76.

Mantoura, R.F.C., A. Dickson, and J.P. Riley, 1978. The compelxation of metals with humic materials in natural waters. Estuarine and coastal Marine Science 6: 387-408.

Mohamed, H.A. 2002. Heavy metals in Suez Canal relevant to the impacts of land based surces. Ph.D. thesis, Fac. Sci., Mans. Univ., Pp. 223.

Mohapatra, S.P. 1988. Distribution of heavy metals in polluted creek sediment. Environment Monitoring and Assessment 10(2): 157-163.

Moore, J.W. 1991. Inorgnaic contaminants of surface water Springer-Verlag New York, Inc. Perlin, Heidelberg, London, Pp. 334.

Morcos, S.A. and J.P. Riley, 1966. Chlorinity, salinity, density and conductivity of seawater from the Suez Canal region. Deep-Sea Research, 13: 741-749.

Nicholsen, R.A. and P.J. Moore, 1981. The distribution of heavy metals in the surficial sediments of the North Sea. Rapp. P.V. Reu. Cons. Int. Explor. Mer., 181: 35-48. 
Rajkumar, W., R. Mungal and V. Bahadoorsingh, 1992. Heavy metal concentration in seawater, sediment and biota (Donax striatus) along the east coast of Trinidad. Carribean Marine Studies, 3: 26-32.

Schindler, P.W. 1976. Heterogenous equilibria involving oxides, hydroxides and carbonates. In R.F. Gould (ed.) Equilibrium contents in natural water systems. Amer. Chem. Soc. Adv. Chemistry Series, 67: 196-221.

Tesier, A., P. Campbell, and M. Bisson, 1979. Sequential extraction for the speciation of particulate trace metals, Annalen der Chemie, 51: 844-851.

UNEP, United Nation Environment Programme, 1997. Vulnerability assessment of the low-lying coastal areas in the southern part of the Suez Canal in the context of climate change sand sea-level rise "Egypt" Pp. 205.

Zirino, A. and S. Yamamoto, 1972. A pH-dependent model for the chemical speciation of $\mathrm{Cu}, \mathrm{Zn}, \mathrm{Cd}$ and $\mathrm{Pb}$ in seawater, Limnology and Oceanography, 17: 661-671.

(Received: 10 June, 2004) 